Laser Chem., 1999, Vol. 19, pp. 253-262

Reprints available directly from the publisher Photocopying permitted by license only
(C) 1999 OPA (Overseas Publishers Association) N.V.

Published by license under the Harwood Academic Publishers imprint, part of The Gordon and Breach Publishing Group.

\title{
FEMTOSECOND INFRARED STUDIES OF CHEMICAL BOND ACTIVATION
}

\author{
M. C. ASPLUND, H. YANG, K. T. KOTZ, S. E. BROMBERG, \\ M. J. WILKENS and C. B. HARRIS* \\ Department of Chemistry, University of California, Berkeley, \\ CA 94720, USA
}

(Received 22 October 1997)

\begin{abstract}
The identification of the intermediates observed in bond activation reactions involving organometallic complexes on time scales from femtoseconds to milliseconds has been accomplished through the use of ultrafast infrared spectroscopy. $\mathrm{C}-\mathrm{H}$ bond activation by the molecule $\mathrm{Tp} \mathrm{p}^{*} \mathrm{Rh}(\mathrm{CO})_{2}$ showed a final activation time of $200 \mathrm{~ns}$ in cyclic solvents, indicating a reaction barrier of $8.3 \mathrm{kcal} / \mathrm{mol}$. An important intermediate is the partially dechelated $\eta 2-\mathrm{Tp}^{*} \mathrm{Rh}(\mathrm{CO})(\mathrm{S})$ solvent complex, which was formed $200 \mathrm{ps}$ after the initial photoexcitation. $\mathrm{Si}-\mathrm{H}$ bond activation by $\mathrm{CpM}(\mathrm{CO})_{3}(\mathrm{M}=\mathrm{Mn}, \mathrm{Re})$ showed some product formation in less than $5 \mathrm{ps}$, indicating that the $\mathrm{Si}-\mathrm{H}$ activation reaction is barrierless. The activated product was formed on several timescales, from picoseconds to nanoseconds, suggesting that there are different pathways for forming final product which are partitioned by the initial photoexcitation.
\end{abstract}

Keywords: Organometallic complexes; femtosecond infrared

\section{INTRODUCTION}

The discovery of organometallic complexes which break, or activate, $\mathrm{C}-\mathrm{H}$ and $\mathrm{Si}-\mathrm{H}$ bonds has led to significant study of these complexes in an attempt to understand their unique chemical reactivity. These reactions are interesting for their uses in chemical synthesis, but also because they are model bimolecular reactions and can therefore provide insight into more general principles of chemical reactivity. In this study we have been able to address significant

\footnotetext{
*Corresponding author.
} 
questions concerning the mechanisms of these reactions, identifying key intermediates and the dynamics leading between them.

Previous attempts to study these precesses $[1,2]$ have focused on variety of reaction media, including gas phase, unreactive liquid noble gases, low temperature glasses and matrix isolation. In each of these cases, the reaction is simplified and/or slowed relative to that which takes place in room temperature alkane or silane solution, a fact which has allowed for appreciable insight into the physical system. Since the reaction depends critically on interactions with the solvent, however, these studies do not allow probing of the underlying chemistry. The development of femtosecond infrared probe techniques has allowed us to probe the actual chemistry in the most relevant environment, and to do so in real time [3-5].

\section{EXPERIMENTAL TECHNIQUES}

\section{Ultrafast Measurements}

Ultrafast infrared measurements were made using a home built amplified Ti:Saphire laser which has been previously described in the literature [6]. Briefly, it consists of a Ti:saphire oscillator which produces $40 \mathrm{fs}$ pulses at $80 \mathrm{MHz}$. This output is amplified in a transversely pumped Bethune dye cell, using the $532 \mathrm{~nm}$ second harmonic output of a $30 \mathrm{~Hz}$ Spectra-Physics YAG laser to give $10 \mu \mathrm{s}$ pulses at $800 \mathrm{~nm}$. This amplified output is recompressed to $<100 \mathrm{fs}$ and is split into three parts. The first part is used to generate white light continuum, the $590 \mathrm{~nm}$ portion of the continuum amplified to $400 \mu \mathrm{J}$ and frequency doubled in a BBO crystal to make $295 \mathrm{~nm}$ U.V. light. This pulse is used to photoinitiate the reaction. The second part is also used to generate white light continuum, from which the $690 \mathrm{~nm}$ part is amplified. The remaining $800 \mathrm{~nm}$ light is amplified and mixed with the $690 \mathrm{~nm}$ light in a lithium iodate difference frequency crystal to form the femtosecond IR pulses from 1800 to $2100 \mathrm{~cm}^{-1}$. This infrared light is separated into probe and reference portions, which are subsequently passed through a monochrometer and then into separated detectors. All measurements are made with magic angle polarization.

The sample was prepared to give a solution which had an absorbance of 0.6 at the pump wavelength $(295 \mathrm{~nm})$. It was then 
deoxygenated by bubbling $\mathrm{N}_{2}$ gas through it for 10 minutes, and sealed to exclude water vapor and oxygen. The sample was flowed between $4 \mathrm{~mm}$ thick $\mathrm{CaF}_{2}$ windows at a rate fast enough to ensure that a fresh sample was encountered at each laser shot. Sample integrity was monitored by visible and IR static spectroscopy.

\section{Step Scan FTIR}

While ultrafast measurements are useful in understanding the reaction mechanism, many of the important steps in the reaction take place on a much longer time scale. In the present work, final activated products are not fully generated within 1 ns (the maximum time delay of our ultrafast IR system). In order to study reaction dynamics on a longer time scale, we have performed measurements using Step Scan Fourier Transform Infrared Spectroscopy (SS-FTIR).

The instrument employed [7] is a Bruker IFS 88 dual beam FTIR spectrometer, fitted with a modified interferometer driver and fast IR detectors and electronics. Rather than continuously scanning the moving mirror in the interferometer as is done in conventional FTIR, the mirror is moved in a number of discreet steps and data is taken at each step. A Spectra Physics YAG is used to photoinitiate the reaction at $355 \mathrm{~nm}$, and a full time trace is collected with each laser pulse. Each time trace is made up of $m$ points spaced by the smallest time resolution allowed by the digitizer. For these experiments $m=100$ points, and the digitizer takes points every $25 \mathrm{~ns}$, giving us $2.5 \mu \mathrm{s}$ per scan. At each mirror position, 30 pulses are averaged to give improved signal to noise. After data collection, an interferograms corresponding to each time slice is constructed from the time traces, and the interferograms are fourier transformed to give full frequency dependent absorption spectra at each time slice. This method allows us to take full spectra, in this case from 1600 to $2200 \mathrm{~cm}^{-1}$ at 10 or $25 \mathrm{~ns}$ per time slice with good signal to noise.

\section{C-H ACTIVATION BY Tp* Rh(CO) $)_{2}$ ALKANE SOLUTION}

The study of $\mathrm{C}-\mathrm{H}$ bond activating compounds has focused primarily on the $\mathrm{CpM}(\mathrm{CO})_{2}$ system, where $\mathrm{Cp}=$ cyclopentadiene and $\mathrm{M}=\mathrm{Rh}$ or 
Ir. Recently Lees et al. [8] have reported a chemical system, (see Fig. 1) $\mathrm{Tp}^{*} \mathrm{Rh}(\mathrm{CO})_{2}\left(\mathrm{~T}_{\mathrm{p}}^{*}=\mathrm{HB}-\mathrm{Pz}_{3}^{*}, \mathrm{Pz}^{*}=3,5\right.$-dimethylpyrazolyl $)$, which also exhibits $\mathrm{C}-\mathrm{H}$ bond activation chemistry. We have chosen to study this model system for two reasons. First, its $30 \%$ quantum yield is much higher than the $1 \%$ quantum yield of $\mathrm{CpRh}(\mathrm{CO})_{2}$. Second, it contains $\mathrm{CO}$ groups, which have a strong IR absorbance, and which exhibit good sensitivity to the electronic environment of the metal atom, making their absorbance an ideal probe of chemical changes taking place at the metal.

Our ultrafast IR absorption measurements of $\mathrm{Tp}^{*} \mathrm{Rh}(\mathrm{CO})_{2}$ in cyclohexane show an instrument limited depletion of the parent carbonyl stretches at 1980 and $2050 \mathrm{~cm}^{-1}$ indicating the formation of a monocarbonyl species (through the loss of one of the carbonyl ligands from the parent molecule) on a time scale of $350 \mathrm{fs}$. This monocarbonyl then complexes with a solvent alkane molecule, and vibrationally cools to give species A which absorbs at $1972 \mathrm{~cm}^{-1}$. The observed cooling time is $20 \mathrm{ps}$, in good agreement with studies of similar systems. A decays away with a time constant of $200 \mathrm{ps}$ to form another monocarbonyl, $\mathbf{B}$, with a CO absorption at $1990 \mathrm{~cm}^{-1}$.

Ultrafast IR measurements show that $\mathbf{B}$ has a lifetime longer than $1 \mathrm{~ns}$, and so it is necessary to measure the course of the reaction over a longer time scale. Step scan FTIR measurements (see Fig. 3) show that in cyclohexane the lifetime of $\mathbf{B}$ is $230 \mathrm{~ns}$, and that it converts cleanly to the final $\mathrm{C}-\mathrm{H}$ activated product, which has a $\mathrm{CO}$ absorption at $2032 \mathrm{~cm}^{-1}$. The spectrum of $\mathbf{B}$ provides evidence that it is a monocarbonyl (because there is only one CO stretch) and has less
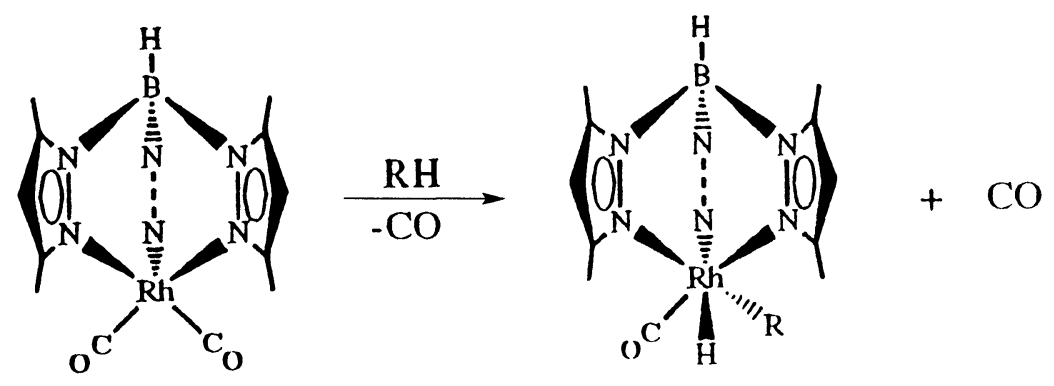

FIGURE $1 \mathrm{C}-\mathrm{H}$ reaction used in this study. 


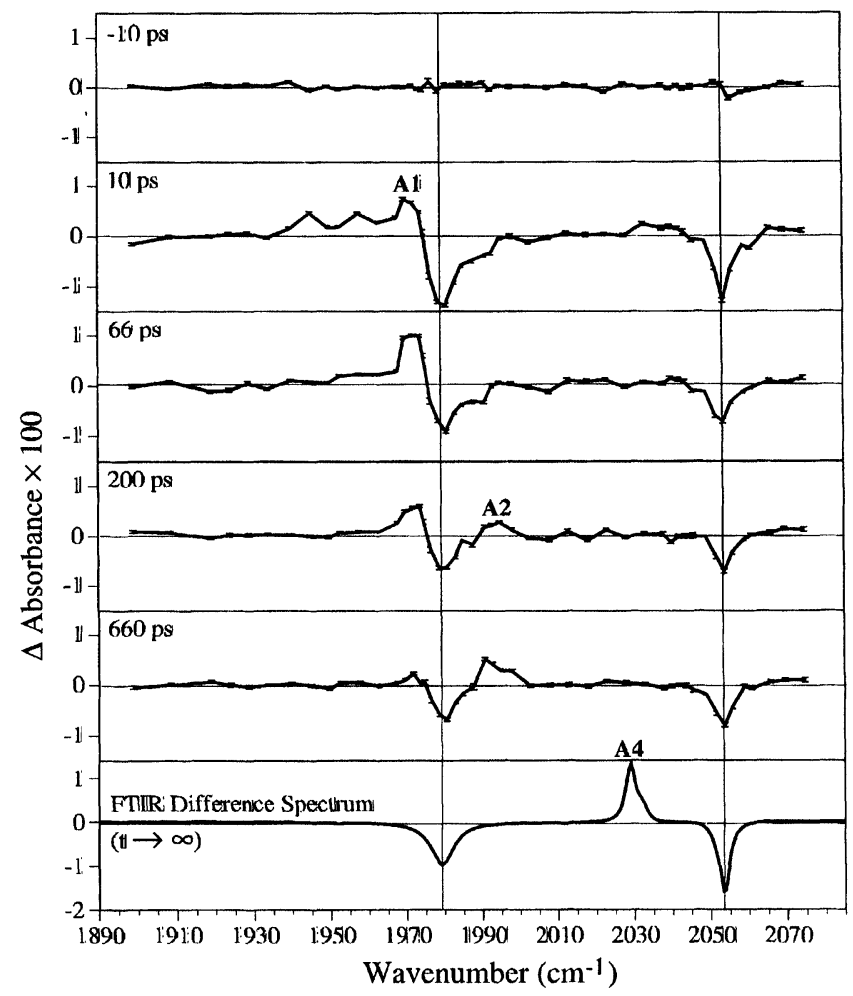

FIGURE 2 Ultrafast IR spectrum of the reaction of $\mathrm{Tp}^{*} \mathrm{Rh}(\mathrm{CO})_{2}$ in cyclohexane.

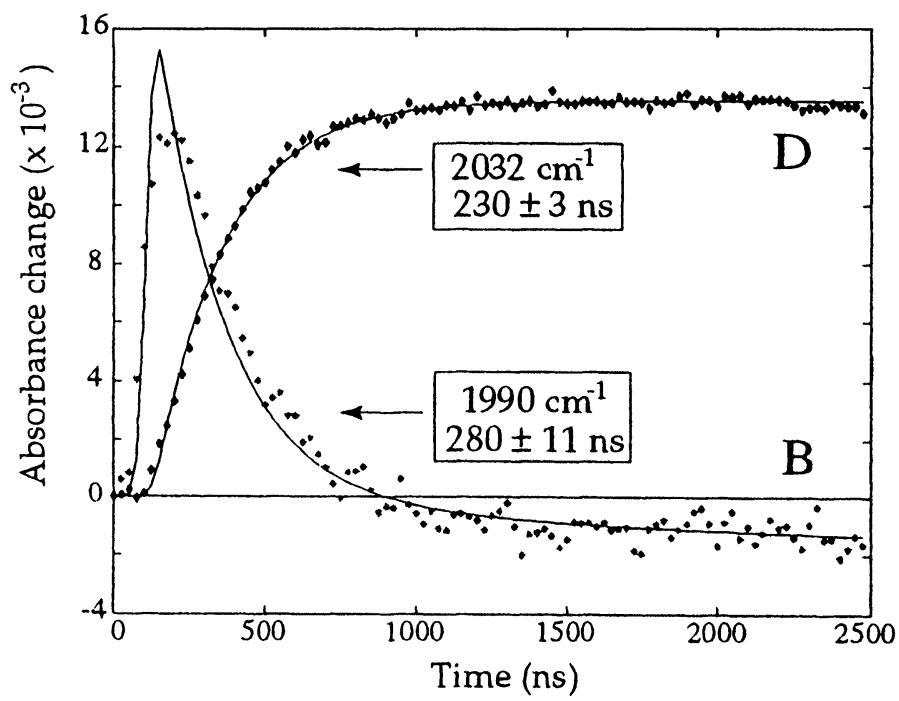

FIGURE 3 Nanosecond IR kinetics for intermediate $\mathbf{B}$ and final product $\mathbf{D}$. 
electron density on the metal center than $\mathbf{A}$ (because the $\mathrm{CO}$ absorption in $\mathbf{A}$ is blue shifted relative to $\mathbf{B}$ ).

In the dicarbonyl ground state, there is evidence that the $\eta^{3}-\mathrm{Tp}^{*} \mathrm{Rh}(\mathrm{CO})_{2}$ is in equilibrium with $\eta^{2}-\mathrm{Tp}^{*} \mathrm{Rh}(\mathrm{CO})_{2}$, with an equilibrium constant $K_{\mathrm{eq}}=100$ favoring the $\eta^{3}$ species. Results of recent density functional theory calculations by Zaric and Hall indicate that the closely related monocarbonyl complex $\mathrm{Tp}^{*} \mathrm{Rh}(\mathrm{CO})\left(\mathrm{CH}_{4}\right)$ may be more stable in an $\eta^{2}$ form than in the $\eta^{3}$ form. Thus it is likely that $200 \mathrm{ps}$ after photexcitation, the intermediate A dechelates to form an $\eta^{2}-\mathrm{Tp} \mathrm{p}^{*} \mathrm{Rh}(\mathrm{CO})$ (cyclohexane) complex.

A model chemical system for probing the effect of dechelation on $\mathrm{CO}$ frequency and for establishing the identity of $\mathbf{B}$ is the $\eta^{2}$ complex $\mathrm{Bp}^{*} \mathrm{Rh}(\mathrm{CO})_{2}\left(\mathrm{Bp}^{*}=\mathrm{H}_{2} \mathrm{~B}-\mathrm{Pz}_{2}^{*}, \mathrm{Pz}^{*}=3,5\right.$-dimethylpyrazolyl). This complex, with only two pyrazolyl rings, is known to be a stable $\eta^{2}$ species.

Photoinduced loss of $\mathrm{CO}$ from $\mathrm{Bp}^{*} \mathrm{Rh}(\mathrm{CO})_{2}$ should produce a monocarbonyl species very similar to the $\eta^{2}-\mathrm{Tp}{ }^{*} \mathrm{Rh}(\mathrm{CO})$ species. Static IR and NMR studies show that there is no $\mathrm{C}-\mathrm{H}$ bond activation by the $\mathrm{Bp}^{*}-\mathrm{Rh}(\mathrm{CO})$ complex upon photolysis, suggesting that the $\eta^{2}-\mathrm{Tp}^{*} \mathrm{Rh}(\mathrm{CO})$ complex could not activate $\mathrm{C}-\mathrm{H}$ bonds. Time resolved IR studies in the femtosecond and nanosecond domain show that a monocarbonyl peak at $1990 \mathrm{~cm}^{-1}$ is present immediately following excitation, and that this peak remains throughout the observed time period ( $>5 \mu \mathrm{s}$ ). This confirms the identity of the intermediate B as an $\eta^{2}-\mathrm{Tp}^{*} \mathrm{Rh}(\mathrm{CO})(\mathrm{H})(\mathrm{R})$ complex. From the reactivity of $\mathrm{Bp}^{*}$ we know that in order to stabilize the final alkyl hydride product, the complex must rechelate to form the $\eta^{3}-\mathrm{Tp}^{*} \mathrm{Rh}(\mathrm{CO})(\mathrm{H})($ alkyl) product.

We now have a picture of the entire mechanism of this reaction, from the photo-dissociation and solvation steps in the first few hundred femtoseconds, through the vibrational cooling and dechelation steps in the next 200 picoseconds, and finally to the breaking of the $\mathrm{C}-\mathrm{H}$ bond, and formation of the metal alkyl hydride complex after 200 nanoseconds. We can also infer reaction barriers from the time scales to give a full picture of both structures and processes. This data is summarized in Figure 4. 


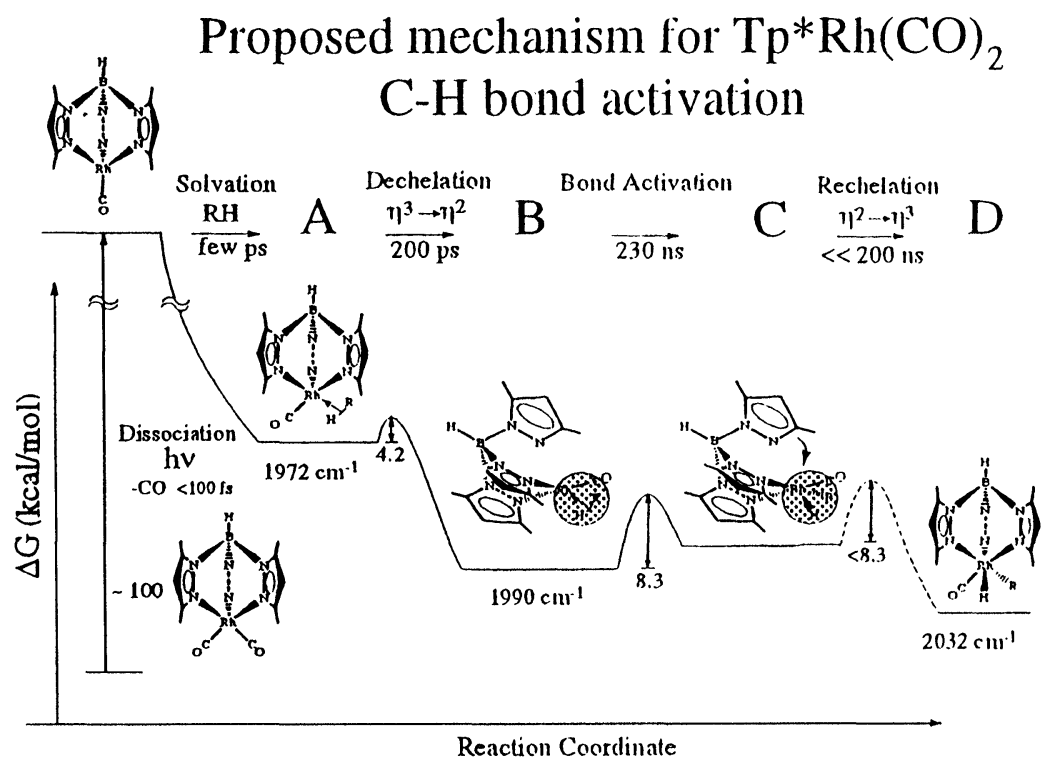

FIGURE 4 Reaction mechanism for $\mathrm{C}-\mathrm{H}$ activation by $\mathrm{Tp}^{*} \mathrm{Rh}(\mathrm{CO})_{2}$.

\section{$\mathrm{Si}-\mathrm{H}$ BOND ACTIVATION}

A system which has many interesting similarities to the $\mathrm{C}-\mathrm{H}$ bond activation is the analogous process involving $\mathrm{Si}-\mathrm{H}$ bond cleavage. $\mathrm{Si}-\mathrm{H}$ bonds are isoelectronic with $\mathrm{C}-\mathrm{H}$ bonds, and might be expected to display similar chemical properties, but they are weaker and therefore much more reactive. Photochemical reactions of silanes with $\mathrm{CpMn}(\mathrm{CO})_{3}$ and $\mathrm{CpRe}(\mathrm{CO})_{3}$ which are not able to activate, or break, $\mathrm{C}-\mathrm{H}$ bonds in alkanes, are known to activate $\mathrm{Si}-\mathrm{H}$ bonds in silane compounds. Such reactions are interesting and important for reasons similar to those which motivate the study of $\mathrm{C}-\mathrm{H}$ bond cleavage by $\mathrm{Tp}^{*} \mathrm{Rh}(\mathrm{CO})_{2}$, namely the key role that silanes play in large sectors of synthetic chemistry and industrial processes.

It is commonly accepted that with $(\mathrm{Et})_{3} \mathrm{SiH}$ the first step in the photoreaction of $\mathrm{CpMn}(\mathrm{CO})_{3}$ is the photodetachment of one of the $\mathrm{CO}$ ligands [9], producing a coordinatively unsaturated dicarbonyl species, which is solvated to form $\mathrm{CpMn}(\mathrm{CO})_{2}\left(\mathrm{HSiEt}_{3}\right)$. We observe a 


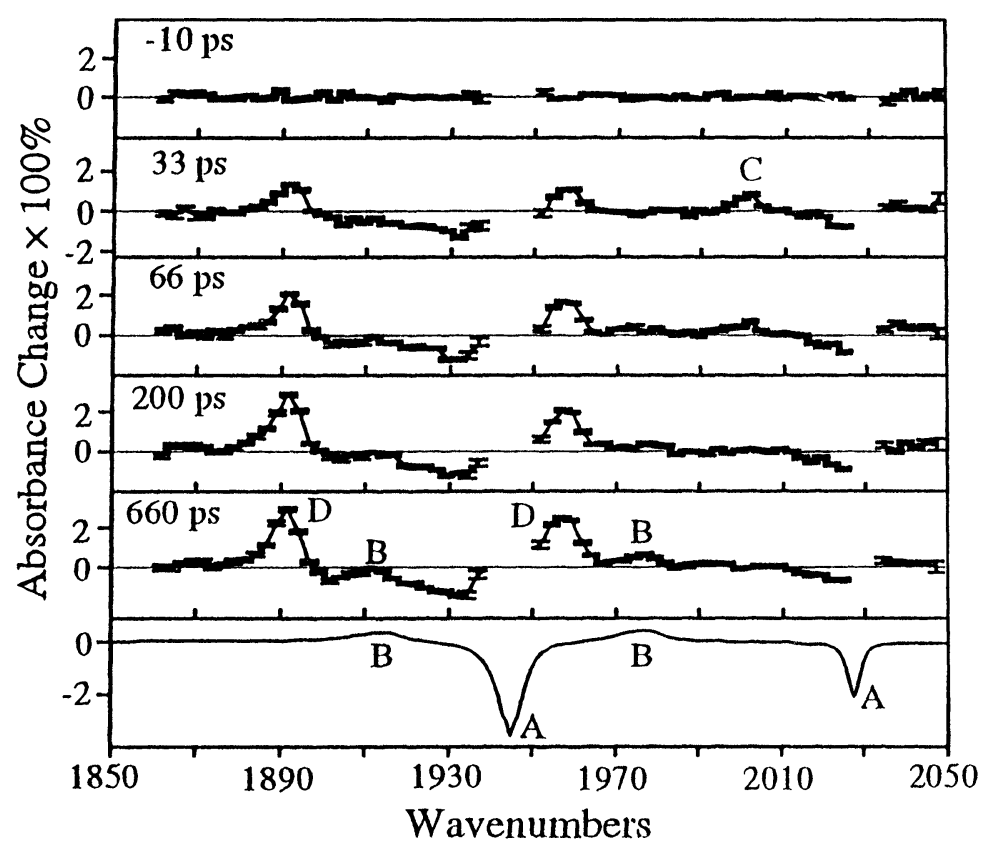

FIGURE 5 Femtosecond IR spectra for $\mathrm{CpMn}(\mathrm{CO})_{3}$.

depletion time [4] for the bleach (Fig. 5, A) of the parent species at $2028 \mathrm{~cm}^{-1}$ which is $<100 \mathrm{fs}$, representing photodissociation of the CO fragment. This parent bleach recovers on a time scale of $32 \mathrm{ps}$, which represents vibrational cooling of non-dissociated parent molecules.

Dicarbonyl species which do not recombine with $\mathrm{CO}$ are rapidly solvated by the $\mathrm{Et}_{3} \mathrm{SiH}$ solvent, giving rise to new bands in the absorption spectrum. Our data show CO absorption peaks at 1892 and $1957 \mathrm{~cm}^{-1}$, which represent $\mathrm{CpMn}(\mathrm{CO})_{2}$ solvated by the ethyl group of the $\mathrm{Et}_{3} \mathrm{SiH}$ (see Fig. 6) This species has been observed before in low temperature glass studies, and is well understood. The peak grows in with a biexponential rise with $\tau=23 \pm 6.4$ and $89 \pm 20 \mathrm{ps}$. The first time constant is associated with vibrational cooling of the initially excited solvent complex.

At early times we also observe a peak at $2002 \mathrm{~cm}^{-1}$ which grows in $23 \pm 2.3 \mathrm{ps}$ and decays away in $90 \pm 6.7 \mathrm{ps}$ (Fig. 4, C). This species is another solvated dicarbonyl which subsequently converts into the alkane complex $\mathbf{D}$ on a time scale of $90 \mathrm{ps}$. The fact that this other 


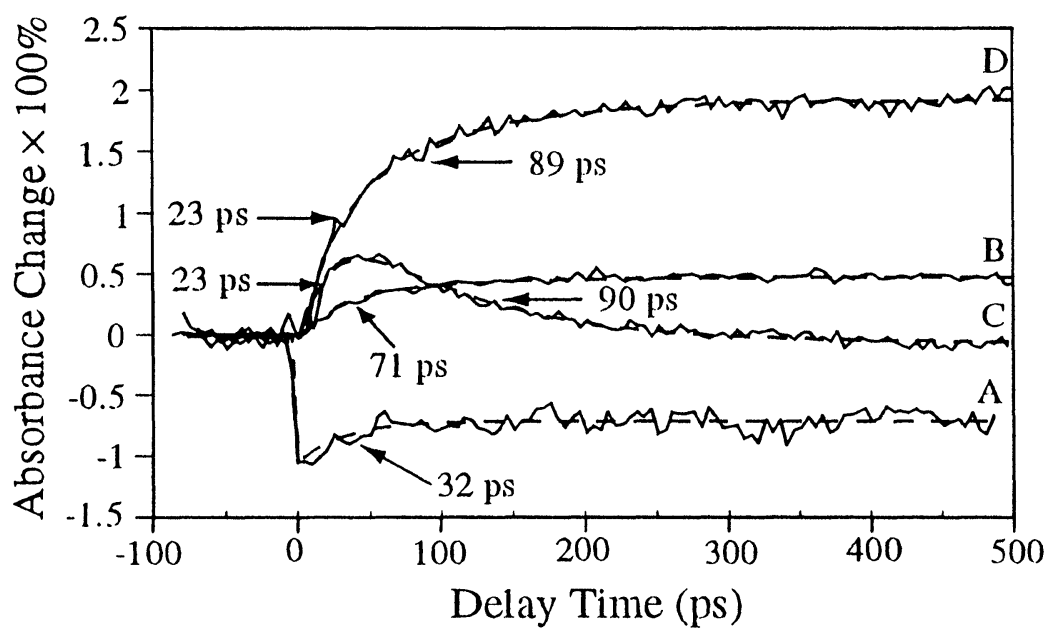

FIGURE 6 Femtosecond IR kinetics for $\mathrm{Si}-\mathrm{H}$ activation by $\mathrm{CpMn}(\mathrm{CO})_{3}$.

intermediate is involved makes the reaction quite interesting. There is a partitioning of intermediates at very early times into two different reactive channels, both of which yield the same product at sufficiently long times ( $>100 \mathrm{ps}$ ).

The identity of the intermediate $\mathbf{C}$ is difficult to assign. We know from previous work that a monocarbonyl is not likely, since only single ligand disociation has been seen in solution phase. We assume that $\mathbf{C}$ is a dicarbonyl, and that the second $\mathrm{CO}$ band is present, but obscured by the intense parent bleach. The $\mathrm{CO}$ stretch is shifted $45 \mathrm{~cm}^{-1}$ to the red of $\mathbf{D}$, we see two possibilities. The first possibility is that this represents another electronic state, possibly a triplet state. The second is that this system is showing reactivity similar to the $\mathrm{Tp}^{*} \mathrm{Rh}(\mathrm{CO})_{2}$ system, and that the reaction proceeds through a dechelation step. Further experiments and $a b$ initio calculations are underway to clarify the identity of $\mathbf{C}$.

On a longer time scale ( $>1 \mathrm{~ns}$ ), we know that $\mathbf{D}$ must convert into the final $\mathrm{Si}-\mathrm{H}$ activated product, since the alkane complex is not present in static difference spectra. This rearrangement is most likely due to a dissociative mechanism, since it takes place on such a long time scale. In this mechanism, $\mathrm{Et}_{3} \mathrm{SiH}$ thermally dissociates, allowing 


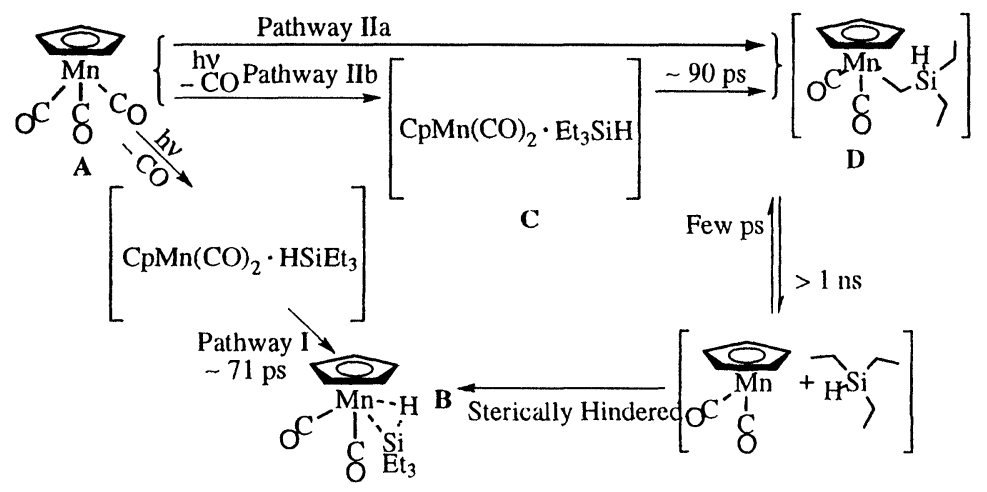

FIGURE 7 Reaction mechanism for $\mathrm{Si}-\mathrm{H}$ activation by $\mathrm{CpMn}(\mathrm{CO})_{3}$.

the metal center to interact with the $\mathrm{Si}-\mathrm{H}$ bond of other solvent molecules. The minimum activation energy would be dictated by the complexation enthalpy of a $\mathrm{CpMn}(\mathrm{CO})_{2}$ and an alkyl group, which is estimated to be $8 \mathrm{kcal} / \mathrm{mol}$. At room temperature this barrier hight corresponds to a reaction time scale of $120 \mathrm{~ns}$.

A tentative overall reaction scheme is shown in Figure 7. After photo-initiation, the reaction proceeds by two different intermediates. The partitioning of these two different pathways is decided in the first $100 \mathrm{fs}$ as the newly formed dicarbonyl is solvated by either a $\mathrm{C}-\mathrm{H}$ bond on an ethyl group or by the $\mathrm{Si}-\mathrm{H}$ bond.

\section{References}

[1] Arndsten, B. A., Bergman, R. G., Mobley, R. A. and Peterson, T. H. (1995). Acc. Chem. Res., $28,154$.

[2] Hill, R. H. and Wrighton, M. S. (1987). Organometallics, 6, 632.

[3] Bromberg, S. E., Yang, H., Asplund, M. C., Lian, T., McNamara, B. K., Kotz, K. T., Yeston, J. S., Wilkens, M., Frei, H., Bergman, R. G. and Harris, C. B. (1997). Science, 278, 260.

[4] Yang, H., Kotz, K. T., Asplund, M. C. and Harris, C. B. (1997). J. Am. Chem. Soc., $119,9564$.

[5] Lian, T., Bromberg, S. E., Yang, H., Proulx, G., Bergman, R. G. and Harris, C. B. (1997). J. Am. Chem. Soc., 118, 2069.

[6] Lian, T., Bromberg, S. E., Asplund, M. C., Yang, H. and Harris, C. B. (1996). J. Phys. Chem., 100, 11994.

[7] Sun, H. and Frei, H. (1997). J. Phys. Chem. B, 101, 205.

[8] Purwoko, A. A., Tibensky, S. D. and Lees, A. J. (1996). Inorg. Chem., 35, 7049.

[9] Young, K. M. and Wrighton, M. S. (1989). Organometallics, 8, 1063. 